\title{
Study of Fault Location Algorithm for Distribution Network with Distributed Generation based on IGA-RBF Neural Network
}

\author{
Huanxin Guan, Ganggang Hao and Hongtao Yu \\ Shenyang Institute of Engineering, Shenyang, China \\ huanxin-guan@163.com
}

\begin{abstract}
The access of the Distributed Generation (DG) has to make the fault location problem of distribution network extremely complex and affect the efficiency and accuracy of fault location. According to the fault information that the Supervisory Control And Data Acquisition (SCADA) of distribution network upload and considering the change of configuration and logic relation of distribution network protection after DGs access, this paper proposed a radial basis function (RBF) neural network based on Improved Genetic Algorithm (IGA), which used the real-coded genetic algorithm with adaptive crossover and mutation into the gradient-dropping algorithm as the RBF network learning algorithm, and constructed a new switch function and fitness function. And then the improved algorithm was applied to the fault location of distribution network containing distributed power supply. The simulation results show that the RBF neural network based on IGA not only has the advantages of simple structure and fast operation, but also has better generalization performance. The analysis and comparison results show that the optimized Improved algorithm can effectively improve the convergence speed and precision, and it has good fault-tolerance to the lack or distortion of the fault information.
\end{abstract}

Keywords: Distributed generation; distribution network; fault location; IGA-RBF neural network; gradient-dropping algorithm; self-adaptive

\section{Introduction}

In the current distribution network automation system at home and abroad, the feeder terminal unit (FTU) and other devices are widely used for distribution system fault location to provide a richer remote communication and remote measure data. With the development of communication technology and computer technology, the feeder automation technology is also increasingly intelligent, integrated, and now advanced feeder terminal device FTU integrated remote metering, remote communication, remote control, fault monitoring and many other features. So the main station remote FTU mode has become the mainstream of the development of distribution automation system. Under this situation, the research on the fault location method of the distribution network with the full use of FTU information is booming, and the method to solve the fault location mainly includes matrix method ${ }^{[2,3]}$, expert system ${ }^{[5]}$, genetic algorithm ${ }^{[6]}$, neural network method ${ }^{[7]}$, etc.

The information sources used in distribution network fault location mostly come from the outdoor FTU, which is influenced by weather, electromagnetic interference and other factors. So FTUs upload information may appear distorted. The method proposed by reference ${ }^{[2]}$ is more accurate to the information required to upload. Although the matrix algorithm is intuitive and fast, it has poor fault tolerance to the fault information. The neural network has a strong self-learning ability, nonlinear mapping ability and fault tolerance, which has been widely applied for fault detection and fault location of power system, but its results of the training are not stable and easily fall into local optima. The genetic algorithm is a global optimization algorithm based on the genetic mechanism of 
human beings. It can solve the problem of fault diagnosis from the perspective of global optimization, which has a high fault tolerance, and can get the global optimal solution. This paper proposes the RBF neural network method based on improved genetic algorithm, the fault information provided by FTU is used as the input of which, that is simple and has a certain fault tolerance. ${ }^{[8]}$

\section{Distribution Network Containing Distributed Generation}

\subsection{The Influence of Distributed Generation Access on Fault Location}

Traditional distribution network is generally implemented by a single power supply. The basic principle of the past algorithm for fault location of single power supply radial distribution network is: the fault section is located between the last FTU monitoring points which have experienced the fault current and the first FTU monitoring point of the feeder terminal unit without fault. With the large number of DGs access to distribution network, it has many effects on the distribution network. In the structure, it increases new network branches, and enhances the complexity of the network topology. in function, distribution network does not only absorb the power of the DGs but also carry out the allocation. in the operation, distribution network still withstands the instability of the DGs. The access of DGs changes the flow distribution on the line. When the load is very small, the output power of DG is likely to flow back to the system side. In this case, some of the feeder segment will become double-ended power supply. This paper sets up that the positive direction is from the system power to the end of the line, the system power supply side for the upstream, the end of the line for the downstream. Taking the $K 1$ part of system malfunctioned as an example, as shown in Figure 1. When the system $K 1$ is in trouble, the FTU monitoring points 1 and 2 can detect the fault current, but the fault location cannot be judged via the previous fault location algorithm.

The closed-loop operation mode of distribution network is used for the line of higher reliability requirement and should be segmented to run. When the $K 1$ is in trouble, 9,10 , 7.8, 12 FTU monitoring points on feeder L2 would detect fault current, and which is set in the opposite direction of the network, according to the previous fault location method. If the fault occurs at the end of the L1 of the feeder, and the capacity of DG2 is too small, the tidal current direction of the whole distribution network would be not easy to determine, owing to the over-current condition of every FTU monitoring point on the feeder L2 is difficult to determine. The misjudgment may occur between 10 and 12 FTU monitoring points. Therefore, the traditional fault location method of distribution network can not be applied to the fault location of the current multi - power distribution network.

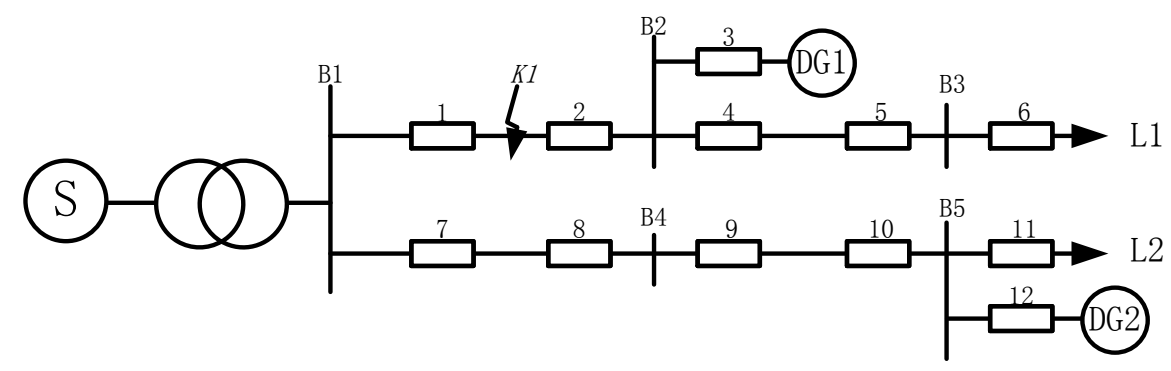

FTU

Figure 1. Typical DG Distribution Network Fault Location Analysis 


\subsection{Fault Function of Distribution Network with Distributed Generation}

Every subsection switch FTU of distribution network has the collection and monitoring function. When the line fails, FTUs will collect the fault state information (over-current, or fault waveform, for the convenience of description, below over-current as an example), in order to realize the fault location the fault current information function must be established from the line fault state to FTU equipment. ${ }^{[13]}$

When the power distribution network is in trouble, the information obtained from FTU is the signal of fault current. In order to realize the fault location, we must establish the conversion from the fault state to the equipment fault current information. This paper assumes that the short circuit capacity of the DG can cover the total load of the system. When the system has a short circuit fault, each of electric source in the distribution network provides the fault current for the short circuit point. The current contains two parts: The first part is the fault current supplied by the main system power supply, namely the path between the main power and the fault points contains the fault current, and the current direction is the positive direction. The second part is the fault current supplied by DGs, namely the path between the DGs and the fault points contains the fault current. Its ultra-limit current direction is determined by the direction coefficient $\varpi$, which may be consistent with fault current direction that the main power supply, and may also be the opposite. The fault function is as follows:

$$
I^{*}\left(x^{j}\right)=\prod_{i=1}^{I} x_{I(i)}^{j}+\sum_{m=1}^{k} \varpi\left(\prod_{n=1}^{N_{m}} x_{N_{m}}^{j}(n)\right)
$$

Where, $I^{*}\left(x^{j}\right)$ is the $j$ th FTU fault function, is the desired state of the switch $j, I$ is the number of FTUs from the main power to the fault point, $k$ represents the number of the DGs, $N_{m}$ is the number of FTUs from the $m$ th to the point of failure, $I(i)$ is the FTU state corresponding to the $i$ th element of the set $I, N m(n)$ is the state corresponding to the $N$ th element of set $\mathrm{Nm}, \varpi$ is the direction coefficient of the current.

\section{RBF Neural Network Theory}

The neural network has the ability of feature extraction and pattern recognition, and can extract the waveform characteristics of different branches accurately to realize fault discrimination. According to the identification of the current state pattern near the fault point, the fault section is judged by the optimization training.

\subsection{RBF Neural Network Structure}

Radial basis function (RBF) network is a strong feed forward neural network of biological background, is widely used in pattern recognition, function approximation, signal processing and control and other fields. RBF network is a three layer network, as shown in Figure 2. The first layer is the input layer, which is composed of the signal source node. The second layer is the hidden layer, the node number is of hidden layer determined by the description problem, and the transformation function of the neuron in the hidden layer is that the radial basis function is radial symmetric center and non-negative nonlinear attenuation function. The third layer is the output layer, and the output of the network is linear weighted of the hidden unit output, which responds to the input mode. ${ }^{[14]}$ 


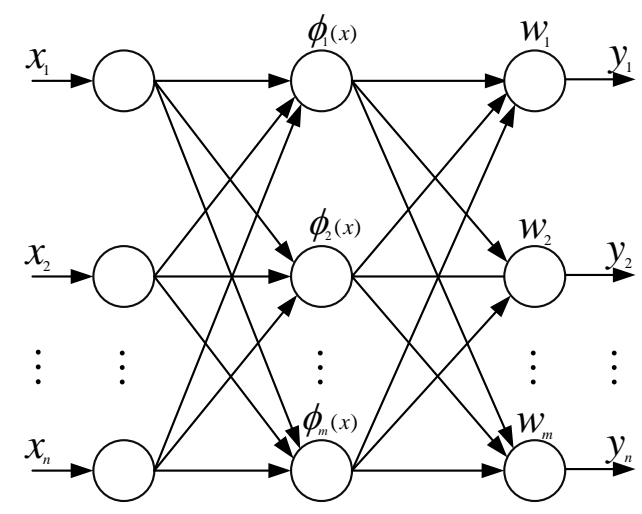

Figure 2. Structure of RBF Neural Network

The Gauss function is commonly used by the RBF network basis function, which can be expressed as:

$\phi_{i}(x)=\exp \left[\frac{-\left\|X-c_{i}\right\|^{2}}{2 \sigma_{i}^{2}}\right], i=1,2, \ldots, m$

Where, $\phi_{i}(x)$ for the output of the $i$ th hidden layer node, $X$ is input sample, $X=\left(x_{1}\right.$, $\left.x_{2} \ldots, x_{n}\right)^{\mathrm{T}}, c_{i}$ is the center of the Gauss function in the $i$ hidden layer node, $\sigma_{i}$ is the variable for the $i$ th layer node, called standard constant or base width.

The output of the RBF network is a linear combination of the hidden layer nodes.

$y_{k}=\sum_{i=1}^{m} w_{i k} \phi_{i}(x), k=1,2, \cdots, p$

\subsection{Network Parameters of Learning Algorithm}

The center $c_{i}$ and width $\sigma_{i}$ parameters of RBF network are studied by gradient descent method, for convenient discussion, only one node in the output layer is taken into account, then the type (1) is substituted in the type (2)

$f(x)=\sum_{i=1}^{m} w_{i} \exp \left[\frac{-\left\|x-c_{i}\right\|^{2}}{2 \sigma_{i}^{2}}\right]$

The expected output of the network is $I^{*}(x)$, so the energy function of the network is:

$E=\frac{1}{2} \sum_{j=1}^{n}\left(I^{*}\left(x^{j}\right)-f\left(x^{j}\right)\right)^{2}$

$f\left(x^{j}\right)$ is brought into the above formula.

$E=\frac{1}{2} \sum_{j=1}^{n}\left(I^{*}\left(x^{j}\right)-\sum_{i=1}^{m} w_{i} \exp \left[\frac{-\left\|x-c_{i}\right\|^{2}}{2 \sigma_{i}^{2}}\right]\right)^{2}$

If the sample number is $L$, then

$E=\frac{1}{2} \sum_{j=1}^{n} \sum_{l=1}^{L}\left(I^{*}\left(x_{l}^{j}\right)-\sum_{i=1}^{m} w_{i} \exp \left[\frac{-\left\|x_{l}^{j}-c_{i}\right\|^{2}}{2 \sigma_{i}^{2}}\right]\right)^{2}$ 
then

$$
\xi\left(x_{l}^{j}, c_{i}, \sigma_{i}\right)=I^{*}\left(x_{l}^{j}\right)-\sum_{i=1}^{m} w_{i} \exp \left[\frac{-\left\|x_{l}^{j}-c_{i}\right\|^{2}}{2 \sigma_{i}^{2}}\right]
$$

at last

$$
E=\frac{1}{2} \sum_{j=1}^{n} \sum_{l=1}^{L}\left(\xi\left(x_{l}^{j}, c_{i}, \sigma_{i}\right)\right)^{2}
$$

When the center and width parameter are updated, the $w_{i}$ is constant, then the formula of the center value and width parameter are the following:

$c_{i}(t+1)=c_{i}(t)-\lambda \frac{\partial E}{\partial c_{i}}$
$\sigma_{i}(t+1)=\sigma_{i}(t)-\beta \frac{\partial E}{\partial \sigma_{i}}$

In the formulas (10) and (11), $\lambda$ and $\beta$ are respectively center value and width parameter of learning efficiency. The formula (9) respectively substituted into (10) and (11), after finishing

$$
\begin{aligned}
& c_{i}(t+1)=c_{i}(t)-\frac{\lambda}{2 \sigma_{i}^{2}} \sum_{j=1}^{n} \sum_{l=1}^{L} \xi\left(x_{l}^{j}, c_{i}, \sigma_{i}\right) \cdot \exp \left[\frac{-\left\|x_{l}^{j}-c_{i}\right\|^{2}}{2 \sigma_{i}^{2}}\right]\left(x_{l}^{j}-c_{i}\right) \\
& \sigma_{i}(t+1)=\sigma_{i}(t)-\frac{\lambda}{2 \sigma_{i}^{3}} \sum_{j=1}^{n} \sum_{l=1}^{L} \xi\left(x_{l}^{j}, c_{i}, \sigma_{i}\right) \cdot \exp \left[\frac{-\left\|x_{l}^{j}-c_{i}\right\|^{2}}{2 \sigma_{i}^{2}}\right]\left(x_{l}^{j}-c_{i}\right)^{2}
\end{aligned}
$$

According to the input sample, the weights of output layer can be obtained via the recursive least square method in recognition theory. In this paper, the learning algorithm is based on the connection between the hidden layer and the output layer.

$w_{i k}(l+1)=w_{i k}(l)+\eta\left[I_{k}^{*}-I_{k}(l)\right] \phi_{i}(x)$

Where $I_{k}^{*}$ for the expected output, $l$ for iteration times, $\eta$ for the learning-rate, commonly $0<\eta<2$ for the iterative convergence.

\section{RBF Network Training based on Improved Genetic Algorithm}

The core problem of designing RBF neural network is to determine the value and other parameters of basis function, the number of hidden nodes, and make the target error of the neural network as small as possible, in order to ensure the generalization ability of neural network.

\subsection{Determination of Fitness Function}

The selection of fitness function in genetic algorithm is very important, and the construction of the energy function $E$ is as follows, which makes the optimal solution of the problem:

$E=\frac{1}{2 L} \sum_{j=1}^{L} \sum_{k=1}^{p}\left(t_{j k}-o_{j k}\right)^{2}$

Where, $t_{j k}$ for the expected output of the $j$ th sample of the $k$ th neuron, $o_{j k}$ is the output of $k$ th neuron of RBFNN on the $j$ th sample.

Thus, the fitness function is constructed as follows: 


$$
f=\frac{1}{1+E}
$$

\subsection{Replication and Selection Operation}

In order to ensure the best individual is not ignored, and retain the excellent characteristics of the previous generation group, in this algorithm the previous generation group will be retained to match. For the individual, in order to maintain the stability and the global convergence of the algorithm, the optimal reserved selection mechanism is adopted here. Where the neural network is trained via the genetic algorithm, due to the real-coded mode does not need to treat optimization parameters for encoding and decoding operation, the optimization parameters are directly connected to the form of real-number vector, to improved the convergence and convergence rate of the algorithm. ${ }^{[18]}$

In order to improve the properties of the initial population, a new method of inter cell generation is used, which divides the value of the optimal parameters into a small number of populations, and then randomly generate an initial individual in each cell. Like this, the generated initial individual will be distributed evenly throughout the entire solution space. In order to overcome the shortcomings of the binary encoding, the genetic algorithm uses real - coded schema. Namely, the node number of RBF hidden layer, the center parameter $c_{i}$, and the width parameter $\sigma_{i}$ are compiled into chromosome. The set of these parameters in the network is considered as an individual, and the initial group is generated by a completely random manner in the initial stage. In the end, The individual with the highest fitness in the current solution is copied to the next generation, so that the final result of the genetic algorithm is the highest fitness before. ${ }^{[19]}$

\subsection{Adaptive Crossover Operation}

The crossover use intermediate recombination in real-coded genetic algorithms, and the selection of the crossover probability $P c$ in genetic algorithm has important influence on the behavior and performance of the algorithm. In order to guarantee the quality of the cross, the crossover probability is generated by adaptive method. Namely, the crossover probability is controlled by the evolutionary degree, so the improved adaptive crossover probability is determined by the following formula ${ }^{[20]}$ :

$$
P_{c}= \begin{cases}P_{c 1}-\frac{\left(P_{c 1}-P_{c 2}\right)}{f_{\text {max }}-f_{\text {avg }}}, & f^{\prime} \geq f_{\text {avg }} \\ P_{c 1} & , f^{\prime}<f_{\text {avg }}\end{cases}
$$

Where, $f_{\max }$ is the maximum fitness value of the population, $f_{\text {avg }}$ is the average fitness of each generation group, $f$ is the larger fitness value for the two crossover individuals.

\subsection{Non-uniform Adaptive Mutation Operation}

The variation is carried out for the individual after the optimization of the crossover. To ensure that every point in the solution space can be searched, and the algorithm has the global convergence, the real-encoded non-uniform mutation is used.

In the non-uniform mutation operation from $X=x_{1} x_{2} \cdots x_{k} \cdots x_{1}$ to $X^{\prime}=x_{1}{ }^{\prime} x_{2}^{\prime} \cdots x_{k}^{\prime} \cdots$ $x_{l}^{\prime}$, if the gene value range of change point $x_{k}$ is $\left[U_{\min }^{k}, U_{\max }^{k}\right]$, The new gene value $x_{k}^{\prime}$ can be determined by the following formula ${ }^{[20]}$ : 
$x_{k}^{\prime}=\left\{\begin{array}{l}x_{k}+\Delta\left(t, U_{\text {max }}^{k}-v_{k}\right), \operatorname{random}(0,1)=0 \\ x_{k}-\Delta\left(t, v_{k}-U_{\text {max }}^{k}\right), \operatorname{random}(0,1)=1\end{array}\right.$

$\Delta(t, y)$ is the random number of non-uniform distribution in the $[0, y]$, the expression of $\Delta(t, y)$ is as follows:

$\Delta(t, y)=y\left(1-r^{\left(1-\frac{t}{T}\right) b}\right)$

Where $t$ are the generations of group evolution, $r$ is $[0,1]$ is a random number of uniform distribution. $T$ is the maximum evolutionary algebra, $b$ is the system parameter.

Meanwhile, in order to guarantee the quality of the variation, the modified adaptive mutation probability is adopted, specific expressions for ${ }^{[19,20]}$ :

$$
P_{m}= \begin{cases}P_{m 1}-\frac{\left(P_{m 1}-P_{m 2}\right)\left(f_{\max }-f\right)}{f_{\text {max }}-f_{\text {avg }}}, & f \geq f_{\text {avg }} \\ P_{m 1} & , f<f_{\text {avg }}\end{cases}
$$

Where, $f_{\max }$ is the maximum fitness value of the population, $f_{\text {avg }}$ is the average fitness of each generation group, $f$ is the fitness value of individual variation.

\subsection{Algorithm Implementation}

The structure optimization and parameter learning of RBF network are divided into two stages: training and evolution. Firstly, the randomly generated $n$ individual components use gradient descent method to operate the center and width parameters of the hidden nodes in the network, the method of least squares to train the weights of network. And secondly, the improved genetic algorithm is used to optimize the numbers of hidden nodes to make the basis functions with the least hidden nodes to meet error requirements, and have RBF network of different width parameters through the alternation of the two processes.

In order to solve the problem of structure optimization of RBF network, the Boolean vector $U^{T}=\left(u_{1}, u_{2}, \ldots, u_{M}\right), u_{i}=\{0,1\}$ is Introduced. When $u_{i}=1$ indicates the existence of hidden nodes, on the contrary, $u_{i}=0$ indicates that the hidden nodes are not present. Every Boolean vector correspondingly generates two chromosomes: one central parameter chromosomes, one width parameter chromosomes. The central parameter chromosomes $U_{c}^{T}$ and width parameter chromosomes $U_{\sigma}^{T}$ all use real-encoded.

\section{Test System Description and Results Analysis}

Figure 3 is a typical DG distribution network, which contains 27 FTUs, 5 DGs and 9 segment lines, 14 positioning sections. In order to verify the accuracy of the fault location in the DGs input, the influence of the bus and transformer so on are not considered. The data of 80 groups of sample data obtained through the simulation experiment was divided into two parts, which were used for network training and testing. the acquired feature vector is used as the input of the network, and the fault state as the output, which trained IGA algorithm optimized RBF network. Then, the trained network is used to test the recognition sample, and the result is shown in Tab.1. 


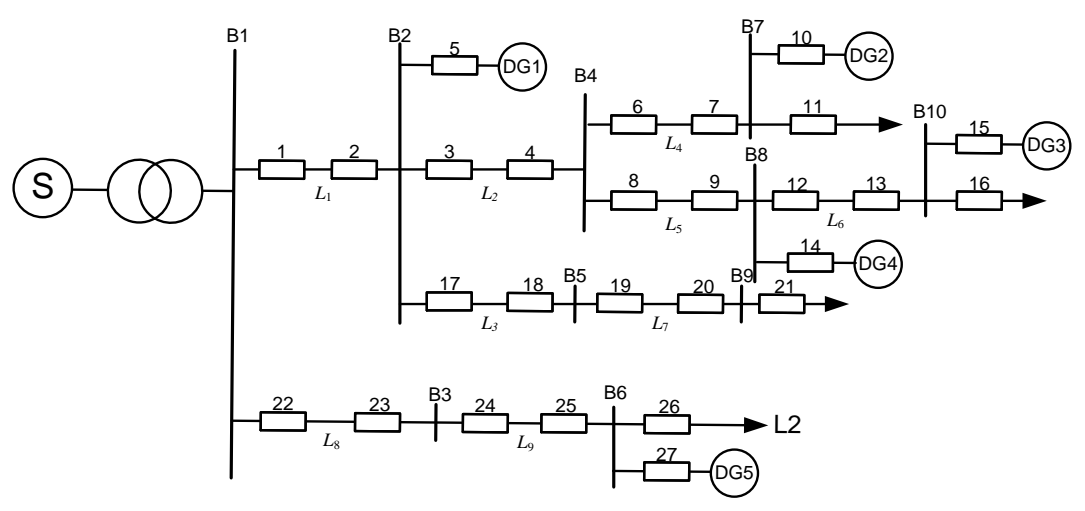

Figure 3. Distribution System with DG in Engineering Practice

In order to verify the validity, fault tolerance and accuracy of the Radial Basis Function neural network based on Improved Genetic Algorithm (IGA-RBFNN) for fault location, in this paper, an example of distribution network has been tested under different conditions. And compared with the results of the traditional RBFNN test used in the literature [22], this test is a total of 30 kinds of clear conditions, some of the results are shown in Table 1 as the test data is more.

Table 1. Results of Fault Location

\begin{tabular}{|c|c|c|c|}
\hline Test number & Fault signal source & $\begin{array}{c}\text { RBF neural network } \\
\text { diagnosis }\end{array}$ & $\begin{array}{c}\text { IGA-RBF neural } \\
\text { network diagnosis }\end{array}$ \\
\hline 1 & No fault signal & No fault & No fault \\
\hline 2 & L3s, F17 & L7 & L7 \\
\hline 3 & L1s, D1,F1,F2,F5 & L1,DG1 & L1,DG1 \\
\hline 4 & $\begin{array}{l}\mathrm{L} 4 \mathrm{~s}, \mathrm{D} 2, \mathrm{~L} 2 \mathrm{~s}, \mathrm{~L} 5 \mathrm{~s}, \mathrm{~F} 3 \\
\mathrm{~F} 8, \mathrm{~F} 10\end{array}$ & L4,L2 & L4 \\
\hline 5 & $\begin{array}{l}\text { L6s,L5s,D4,L4s,F8,F13, } \\
\text { F14,F6F7 }\end{array}$ & $\begin{array}{l}\text { 1D } 6, \mathrm{~L} 4 \\
\text { 2L } 6\end{array}$ & L6,L4 \\
\hline 6 & $\begin{array}{l}\mathrm{L} 5 \mathrm{~s}, \mathrm{~L} 9 \mathrm{~s}, \mathrm{~L} 2 \mathrm{~s}, \mathrm{~L} 6 \mathrm{~s}, \mathrm{D} 4, \mathrm{~F} 3, \\
\mathrm{~F} 13, \mathrm{~F} 14, \mathrm{~F} 24, \mathrm{~F} 25\end{array}$ & $\begin{array}{l}\text { 1D } 5, \mathrm{~L} 9 \\
\text { 2L } 5\end{array}$ & L5,L9 \\
\hline
\end{tabular}

Table, Lis is the main protection of the line $\mathrm{i}$ to send end protection, $\mathrm{D}$ is the protection of the DG, the tests 2-6 represent a different type of fault from a single fault to multiple failures. When the test 2,3 represents the fault protection and the FTU are correct, it can be seen that the two algorithms can accurately locate. There are also different situations of the fault in the test $4-6$, and the improved algorithm can locate the fault accurately. The tests show that the new algorithm can be applied in the fault location of distribution network with DGs, and compared with the results of the two algorithms, The IGA-RBF neural network can accurately determine the fault section in the case of uncertain information, and there was no multi solution and misunderstand the situation. As shown in Figures.4. From the figures, the mean square error (MSE) of the IGA-RBFNN method is significantly decreased and converged in less iteration in contrast with the conventional RBFNN method. Comparing the conventional RBFNN and IGA-RBFNN training performances, it can be said that the IGA-RBFNN takes shorter time to achieve the required training accuracy. 


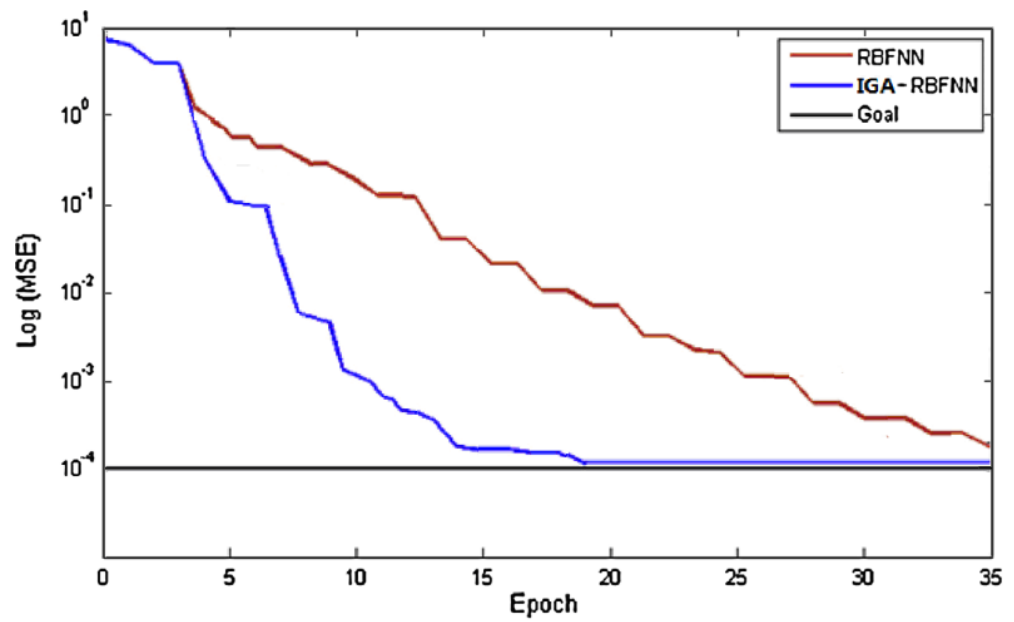

Figure 4. Training Result of IGA-RBFNN and Conventional RBFNN

\section{Conclusion}

The neural network has been successfully applied in the fault location of distribution network, and it has been proved that information is required to be more accurate and its fault tolerance is poor. Compared with the traditional RBF neural network algorithm, This paper took advantage of the advantage that the genetic algorithm optimizes the hidden nodes of RBF network, the center value and width parameter of the basis function. And the algorithm of real-encoded of adaptive mechanism was introduced, the operation of genetic operators was improved to make it mixed with the gradient descent method. The results showed that the proposed fault location method using the IGA-RBFNN can accurately determine the location of faults in a distribution system with several DG units. Compared to the learning process of RBF network, the generalization ability of RBF network is ensured, and the fault location accuracy of distribution network is improved, which makes the learning of RBF rise to a higher level, has a high theoretical significance and practical value in engineering.

\section{Acknowledgments}

This work is supported by National Nature Science Foundation of China under Grant 61304069, 61372195, 61371200 and the Scientific Research Fund of Liaoning Provincial Education Department under Grant L2014517, L2013494, L2012374. I would like to express my gratitude to all those who helped me during the writing of this thesis. I gratefully acknowledge the help of my supervisor, who has offered me valuable suggestions in the academic studies.

\section{References}

[1] J. Q. Wang, S. H. Chen, Y. H. Yin, etc. Fault Region Location Algorithm for Distribution Network Containing Distributed Generation. Electric Power Science and Engineering. 27, 2 (2011)

[2] F. Wang, Y. Sun. an Improved Matrix Algorithm for Fault Location in Distribution Network of Power Systems. Auto Mation of Electric Power Systems. 27, 24 (2003)

[3] J. Q. Yang, Y. T. Chen, L. X. Yang, etc. Study on Improved Matrix Algorithm for Fault Location in Power Distribution Network. High Voltage Engineering. 33, 5(2007)

[4] D. Z. Jiang, F. Zhang, Y. Zhang. Fault Sections Detection and Isolation in Distribution System Based on FTU. Relay. 30, 9 (2002)

[5] J. Fei, Y. D. Shan. Study of Automatic Fault Location System in the Distribution Network. Proceeding of the CSEE. 20, 9 (2000)

[6] H. W. Du, Y. M. Sun, H. J. Liu, etc. Fault Section Diagnosis and Isolation of Distribution Networks Based on Genetic Algorithm. Power System Technology. 24, 5 (2000)

[7] J. Xiao, M. Wang. Compound Algorithm Applied in Fault Location Distribution Network. Automation 
of Electric Power Systems. 24, 8 (2000)

[8] Y. S. Zhou, Y. Q. Zhou, Z. Z. Dai. Algorithm for Fault Section Judgment in Power Distribution Network Based on FTU. Electric Power Automation Equipment. 20, 4 (2000)

[9] Z. N. Wei, H. Ye, Y. P. Zheng. a Refined Genetic Algorithm for the Fault Section Location. Proceedings of the Chinese Society for Electrical Engineering. 22, 4 (2002)

[10] T. S. Yan, D. W. Cui, Y. Q. Tao. Fault Location for Distribution Network by Improved Genetic Algorithm. High Voltage Engineering. 35, 2 (2009)

[11] Z. H. Xu, Y. S. Wang. Study of Fault Region Locating Method for Distribution Networks with Distributed Generators. Power System Protection and Control. 39, 24 (2011)

[12] Y. L. Gao, Y. S. Zhou, P. Peng, R. J. Liu, Z. Z. An. Application of Harmony Algorithm Optimized for Fault Location in Distribution Networks with DG. Power System Protection and Control. 42, 19 (2014)

[13] B. Liu, F. Wang, C. Chen, H. C. Huang, X. Z. Dong. Harmony Search Algorithm for Solving Fault Location in Distribution Networks with DG. Transactions of China Electrotrchnical Society. 28, 5 (2013)

[14] X. M. Yuan, Q. X. Zhuang. Fault Location of Multi-sources Power Distribution Network Based on Neural Network. Coal Mine Machinery. 35, 2 (2014)

[15] J. Q. Wang, S. H. Chen. Fault Location for Distribution Network with Distributed Power Based on Improved Genetic Algorithm. Electric Power Science and Engineering. 29, 12 (2013)

[16] C. Yao, X. D. Liu, B. Zheng. A New Fault Locating Algorithm for Distribution Network with Distributed Gererations. Modern Electric Power. 27, 3 (2010)

[17] L. J. Sun, R. Z. Chen, S. Cai, etc. A New Fault Location Scheme for Distribution System With Distributed Generations. Power System Technology. 37, 6 (2013)

[18] N. Wu, Y. Xu, Y. P. Lu. New Fault Algorithm for Distribution Network with DG. Electric Power Systems. 33, 14 (2009)

[19] W. G. Zhuang, X. J. Shi, J. Tang, Z. C. Xiao. Fault Diagnosis Based on RBFNN Optimized by Improved Genetic Algorithm in Missile Control System. Journal of Central South University (Science and Technology). 42, 1 (2011)

[20] W. G. Zhuang, Y. R. Xu, P. Jiang, X. J. Shi. Application of RBF Neural Network Based on Improved Genetic Algorithm. Journal of Naval Aeronautical and Astronautical University. 25, 3 (2010)

[21] L. Wu, Q. P. Liao, L. Lv, P. Chen. New Approach of Fault Section Location in Distribution Network with DG. Proceedings of the CSU-EPSA. 27, 5 (2015)

[22] Z. Hadi, M. Azah, F. Masoud, M. Marjan. an Optimal Radial Basis Function Neural Network for Fault Location in a Distribution Network with High Penetration of DG Units. Measurement. 46 (2013)

\section{Authors}

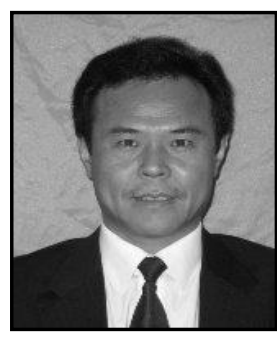

Huanxin Guan received the Ph.D. degree in control engineering from Northeastern University, Shenyang, China, in 2008.

$\mathrm{He}$ is currently a associate professor with the School of Automation, Shenyang Institute of Engineering, Shenyang. His current research interests include fuzzy control theory and fault diagnosis technology.

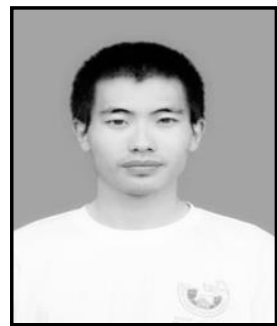

Ganggang Hao received the B.E. degree in Power Engineering from Xuhai college China University of Mining and Technology, Xuzhou, China in 2013.

$\mathrm{He}$ is currently working towards the Master in Electrical Engineering in Shenyang Institute of Engineering. His current research interests include intelligent algorithm, electric power system and intelligent control.

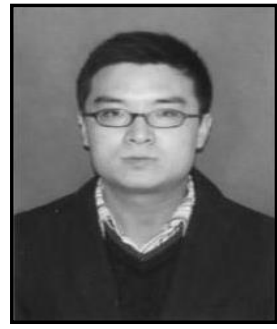

Hongtao Yu received the Ph.D. degree in control engineering from Northeastern University, Shenyang, China, in 2014. $\mathrm{He}$ is currently a lecturer with the School of Automation, Shenyang Institute of Engineering, Shenyang. His current research interests include intelligent optimization theory, neural networks, and intelligent control. 